\title{
A general treatment of alternative expectation formulae
}

\author{
Yang Liu* \\ MRC Biostatistics Unit, University of Cambridge, Cambridge, CB2 0SR, UK
}

\begin{abstract}
The moment of a positive random variable can be obtained by using the survival function. In this paper, we extend existing studies by giving formulae of the expectation of a more general function with respect to a random variable by using either cumulative distribution function or survival function. Both univariate and multivariate examples are given. These formulae can evaluate the expectation when obtaining the probability density/mass function is difficult.
\end{abstract}

Keywords: expectation, singular distribution, cumulative distribution function, survival function, Monte Carlo simulation

\section{Introduction}

The expectation is an important tool to reveal certain characteristics of a random variable. Perhaps the simplest and most frequently used expectation is the mean. In survival analysis, it is well-known that the mean of a non-negative random variable $X$, with its cumulative distribution function $F(x):=\mathbb{P}(X \leq x)$, can be formulated by its survival function $S(x):=\mathbb{P}(X>$ $x)$ as

$$
\mathbb{E}(X)=\int_{0}^{\infty} x d F(x)=\int_{0}^{\infty} S(x) d x
$$

This formula is denoted as an 'alternative formula' for the calculation of the mean. Although the origin of this formula is not clear and we suppose that it has been known and used for some time. Nevertheless, the study of this

\footnotetext{
*Corresponding author

Email address: yang.liu@mrc-bsu.cam.ac.uk (Yang Liu)
} 
alternative formula has raised attention recently. Hong (2012) studied the high order moments

$$
\mathbb{E}\left(X^{n}\right)=\int_{0}^{\infty} n x^{n-1} S(x) d x .
$$

The formula was also studied for non-negative integer-valued random variables (e.g., Lo, 2019; Chakraborti et al., 2019; Song and Wang, 2019a).

Meanwhile, the multivariate version of Eq. (1 and 2) were studied for the non-negative random variable $\left(X_{1}, \ldots, X_{p}\right)$ (e.g., Nadarajah and Mitov, 2003; Hong, 2015; Song et al., 2019). They showed that

$\mathbb{E}\left(X_{1}^{m_{1}} \cdots X_{p}^{m_{p}}\right)=m_{1} \cdots m_{p} \int_{0}^{\infty} \cdots \int_{0}^{\infty} x_{1}^{m_{1}-1} \cdots x_{p}^{m_{p}-1} S\left(x_{1}, \ldots, x_{p}\right) d x_{1} \cdots d x_{p}$,

where $S\left(x_{1}, \ldots, x_{p}\right)$ is the joint survival function of $\left(X_{1}, \ldots, X_{p}\right)$. Similarly, it has been extended to the integer-valued variable (Kwong and Nadarajah, 2018).

More recently, we noticed attempts to generalize these formulae. Besides a specific moment, Lo (2017) studied alternative formulae for the covariance between transformations of two random variables. Song and Wang (2019b) extended these formulae to more general moment generating functions for both continuous and discrete, as well as univariate and multivariate, cases. They showed that, for a non-negative random variable $X$ and $s \in \mathbb{R}$, we have

$$
\mathbb{E}\left(e^{s X}\right)=1+\int_{0}^{\infty} s e^{s x} S(x) d x
$$

and for multivariate $\left(X_{1}, \ldots, X_{p}\right)$ and $\left(s_{1}, \ldots, s_{p}\right) \in \mathbb{R}^{p}$, we have

$$
\mathbb{E}\left(\prod_{i=1}^{p}\left(e^{s_{i} X_{i}}-1\right)\right)=\int_{0}^{\infty} \cdots \int_{0}^{\infty} \prod_{i=1}^{p} s_{i} e^{s_{i} X_{i}} S\left(x_{1}, \ldots, x_{p}\right) d x_{1} \cdots d x_{p}
$$

A recent study further extended it to calculate the expectation of a strictly increasing function (Ogasawara, 2020). However, we believe that these formulae, originated from Eq. (1), can be further extended to calculate the expectation of a more general function. The condition of non-negativity can be relaxed as well. This is particularly important when conducting a Monte Carlo method for the expectation (e.g. importance sampling) is difficult due to the lack of a probability density/mass function. 


\section{Main results and proofs}

We introduce alternative formulae for the univariate variable first, then extend the formulae to the multivariate variable. Before we present main results, we define the lower and upper bound of a random variable. Based on these bounds, we define the absolutely integrable condition with respect to the random variable, which is needed for applying Fubini's theorem.

Definition 1. $s_{1}$ is a real-valued lower bound of a random variable $X$ if $\mathbb{P}\left(X \geq s_{1}+\delta\right)<1$ for all $\delta>0$. $s_{2}$ is a real-valued upper bound of a random variable $X$ if $\mathbb{P}\left(X \leq s_{2}-\delta\right)<1$ for all $\delta>0$.

Definition 2. Given a non-constant function $g(x)$ and a random variable $X$ with a lower bound $s_{1} \in \mathbb{R} \cup\{-\infty\}$ and an upper bound $s_{2} \in \mathbb{R} \cup\{\infty\}$, we say $g(x)$ is absolutely integrable with respect to the random variable $X$ if one of the following holds:

1. Random variable $X$ has a real-valued lower bound $s_{1}$, and

$$
\int_{s_{1}}^{s_{2}} \int_{s_{1}}^{x}|g(t)| d t d F(x)<\infty .
$$

Let $s_{2} \rightarrow \infty$ when $X$ is not upper bounded.

2. Random variable $X$ has a real-valued upper bound $s_{2}$, and

$$
\int_{s_{1}}^{s_{2}} \int_{x}^{s_{2}}|g(t)| d t d F(x)<\infty .
$$

Let $s_{1} \rightarrow-\infty$ when $X$ is not lower bounded.

One typical example when a function $g(x)$ is absolutely integrable with respect to a lower or upper bounded random variable $X$ is that $g(x)$ is bounded and the mean of the random variable $X$ exists. Also, if $X$ has both a real-valued lower bound $s_{1}$ and a real-valued upper bound $s_{2}$, and $g(x)$ is continuous on $\left[s_{1}, s_{2}\right], g(x)$ is absolutely integrable with respect to $X$.

\subsection{Univariate case}

Theorem 1. Suppose $X$ is a random variable whose support is $\left(s_{1}, s_{2}\right) \subsetneq \mathbb{R}$. Let $g(x)$ be a differentiable function whose derivative is absolutely integrable with respect to $X$. 
1. If $s_{1}$ is real-valued, we have

$$
\mathbb{E}(g(X))=g\left(s_{1}\right)+\int_{s_{1}}^{s_{2}} g^{\prime}(x) S(x) d x .
$$

Let $s_{2} \rightarrow \infty$ when $X$ is not upper bounded.

2. If $s_{2}$ is real-valued, we have

$$
\mathbb{E}(g(X))=g\left(s_{2}\right)-\int_{s_{1}}^{s_{2}} g^{\prime}(x) F(x) d x .
$$

Let $s_{1} \rightarrow-\infty$ when $X$ is not lower bounded.

Proof. If $s_{1}$ is real valued, we have

$$
\mathbb{E}\left(g(X)-g\left(s_{1}\right)\right)=\int_{s_{1}}^{s_{2}}\left(g(x)-g\left(s_{1}\right)\right) d F(x)=\int_{s_{1}}^{s_{2}} \int_{s_{1}}^{x} g^{\prime}(t) d t d F(x) .
$$

Since $g^{\prime}(x)$ is absolutely integrable with respect to $X$, by Fubini's theorem, we then have

$$
\mathbb{E}\left(g(X)-g\left(s_{1}\right)\right)=\int_{s_{1}}^{s_{2}} g^{\prime}(t) \int_{t}^{s_{2}} d F(x) d t=\int_{s_{1}}^{s_{2}} g^{\prime}(x) S(x) d x .
$$

Analogously, if $s_{2}$ is real valued, we have

$$
\begin{aligned}
\mathbb{E}\left(g(X)-g\left(s_{2}\right)\right) & =-\int_{s_{1}}^{s_{2}}\left(g\left(s_{2}\right)-g(x)\right) d F(x)=-\int_{s_{1}}^{s_{2}} \int_{x}^{s_{2}} g^{\prime}(t) d t d F(x) \\
& =-\int_{s_{1}}^{s_{2}} g^{\prime}(t) \int_{s_{1}}^{t} d F(x) d t \\
& =-\int_{s_{1}}^{s_{2}} g^{\prime}(x) F(x) d x .
\end{aligned}
$$

Remark 1. Theorem 1 can also be confirmed by using the integration by parts. The support can be relaxed to a set $E \subset\left(s_{1}, s_{2}\right)$ whose Lebesgue measure is $s_{2}-s_{1}$. In the proof, we do not require a random variable $X$ to have a probability density function. In fact, the formulae hold as long as the cumulative distribution function of $X$ is obtainable. 
It is clear that the formula for the moment generating function is a specific case of Eq. (3). In addition to that, since the characteristic function is more powerful for the inference of statistics, we are able to obtain the formula for the characteristic function of a lower or upper bounded random variable provided the mean exists. This is available due to the fact that characteristic function has a bounded derivative. Without loss of generality, for a random variable with a lower bound $s$ and any $t \in \mathbb{R}$, we have

$$
\mathbb{E}\left(e^{i t X}\right)=e^{i t s}+\int_{s}^{\infty} i t e^{i t x} S(x) d x .
$$

This facilitates us to obtain any moment of the random variable.

We now extend Theorem 1 to a unbounded random variable $X$ with a support $(-\infty, \infty)$. Given a subset $E \subset(-\infty, \infty)$, we denote $T_{E}(X)$ as the truncated random variable derived from $X$ after restricting its support to $E$.

Corollary 1. Suppose $X$ is an unbounded random variable with a support $(-\infty, \infty)$. Given a differentiable function $g(x)$, if there is a real-valued number $s$ such that $g^{\prime}(x)$ is absolutely integrable with respect to $T_{(-\infty, s)}(X)$ and $T_{(s, \infty)}(X)$, we have

$$
\mathbb{E}(g(X))=g(s)-\int_{-\infty}^{s} g^{\prime}(x) F(x) d x+\int_{s}^{\infty} g^{\prime}(x) S(x) d x .
$$

PROOF. We divide the expectation by

$$
\begin{aligned}
\mathbb{E}(g(X)-g(s)) & =\int_{-\infty}^{\infty}(g(x)-g(s)) d F(x) \\
& =\int_{-\infty}^{s}(g(x)-g(s)) d F(x)+\int_{s}^{\infty}(g(x)-g(s)) d F(x) \\
& =-\int_{-\infty}^{s} \int_{x}^{s} g^{\prime}(t) d t d F(x)+\int_{s}^{\infty} \int_{s}^{x} g^{\prime}(t) d t d F(x)
\end{aligned}
$$

Since $g^{\prime}(x)$ is absolutely integrable with respect to $T_{(-\infty, s)}(X)$ and $T_{(s, \infty)}(X)$, by Fubini's theorem, we then have

$$
\begin{aligned}
\mathbb{E}(g(X)-g(s)) & =-\int_{-\infty}^{s} g^{\prime}(t) \int_{-\infty}^{t} d F(x) d t+\int_{s}^{\infty} g^{\prime}(t) \int_{t}^{\infty} d F(x) d t \\
& =-\int_{-\infty}^{s} g^{\prime}(x) F(x) d x+\int_{s}^{\infty} g^{\prime}(x) S(x) d x
\end{aligned}
$$


Denote the alternative survival function by $S^{*}(x):=\mathbb{P}(X \geq x)$. For an integer-valued random variable, since its cumulative distribution function is constant between integers, it is straightforward to have:

Corollary 2. Suppose $X$ is an integer-valued random variable with a lower bound $n_{1} \in \mathbb{Z}$ and an upper bound $n_{2} \in \mathbb{Z}$. Given any function $g(x)$, we have

1.

$$
\begin{aligned}
\mathbb{E}(g(X)) & =g\left(n_{1}\right)+\sum_{k=n_{1}}^{n_{2}-1}(g(k+1)-g(k)) S(k) \\
& =g\left(n_{1}\right)+\sum_{k=n_{1}}^{n_{2}-1}(g(k+1)-g(k)) S^{*}(k+1) .
\end{aligned}
$$

Let $n_{2} \rightarrow \infty$ when $X$ is not upper bounded.

2 .

$$
\mathbb{E}(g(X))=g\left(n_{2}\right)-\sum_{k=0}^{n_{2}-n_{1}-1}\left(g\left(n_{2}-k\right)-g\left(n_{2}-k-1\right)\right) F\left(n_{2}-k-1\right) .
$$

Let $n_{1} \rightarrow-\infty$ when $X$ is not lower bounded.

Note that, Eq. (4) is also given in Jones (2019).

\subsection{Multivariate case}

We now extend Theorem 1 to multivariate case.

Theorem 2. Suppose $\left(X_{1}, \ldots, X_{p}\right)$ is a multivariate random variable with a lower bounds $\left(s_{11}, \ldots, s_{1 p}\right)$ and an upper bounds $\left(s_{21}, \ldots, s_{2 p}\right)$. The marginal variable $X_{k}$ has a support $\left(s_{1 k}, s_{2 k}\right) \subsetneq \mathbb{R}, k=1, \ldots, p$. Let $G\left(x_{1}, \ldots, x_{p}\right)=$ $\prod_{k=1}^{p} g_{k}\left(x_{k}\right)$ be a differentiable function when $\prod_{k=1}^{p} g_{k}^{\prime}\left(x_{k}\right)$ is absolutely integrable with respect to $\left(X_{1}, \ldots, X_{p}\right)$.

1. If the survival function $S\left(x_{1}, \ldots, x_{p}\right)$ is continuous at real-valued $\left(s_{11}, \ldots, s_{1 p}\right)$, we have

$\mathbb{E}\left(\prod_{k=1}^{p}\left(g_{k}\left(X_{k}\right)-g_{k}\left(s_{1 k}\right)\right)\right)=\int_{s_{11}}^{s_{21}} \cdots \int_{s_{1 p}}^{s_{2 p}} \prod_{k=1}^{p} g_{k}^{\prime}\left(x_{k}\right) S\left(x_{1}, \ldots, x_{p}\right) d x_{1} \cdots d x_{p}$.

Let $s_{2 k} \rightarrow \infty, 1 \leq k \leq p$, when $\left(X_{1}, \ldots, X_{p}\right)$ is not upper bounded. 
2. If the cumulative distribution function $F\left(x_{1}, \ldots, x_{p}\right)$ is continuous at real-valued $\left(s_{21}, \ldots, s_{2 p}\right)$, we have

$$
\mathbb{E}\left(\prod_{k=1}^{p}\left(g_{k}\left(X_{k}\right)-g_{k}\left(s_{2 k}\right)\right)\right)=(-1)^{p} \int_{s_{11}}^{s_{21}} \cdots \int_{s_{1 p}}^{s_{2 p}} \prod_{k=1}^{p} g_{k}^{\prime}\left(x_{k}\right) F\left(x_{1}, \ldots, x_{p}\right) d x_{1} \cdots d x_{p} .
$$

Let $s_{1 k} \rightarrow-\infty, 1 \leq k \leq p$, when $\left(X_{1}, \ldots, X_{p}\right)$ is not lower bounded.

Proof. We consider the first case. For the second case, the proof is analogous and hence omitted here. We have

$$
\begin{aligned}
& \mathbb{E}\left(\prod_{k=1}^{p}\left(g_{k}\left(X_{k}\right)-g_{k}\left(s_{1 k}\right)\right)\right)=\int_{s_{11}}^{s_{21}} \cdots \int_{s_{1 p}}^{s_{2 p}}\left(\prod_{k=1}^{p}\left(g_{k}\left(x_{k}\right)-g_{k}\left(s_{1 k}\right)\right)\right) d F\left(x_{1}, \ldots, x_{p}\right) \\
& =\int_{s_{11}}^{s_{21}} \cdots \int_{s_{1 p}}^{s_{2 p}} \prod_{k=1}^{p} \int_{s_{1 k}}^{x_{k}} g_{k}^{\prime}\left(t_{k}\right) d t_{k} d F\left(x_{1}, \ldots, x_{p}\right) \\
& =\int_{s_{11}}^{s_{21}} \cdots \int_{s_{1 p}}^{s_{2 p}} \prod_{k=1}^{p} g_{k}^{\prime}\left(t_{k}\right) \int_{t_{1}}^{s_{21}} \cdots \int_{t_{p}}^{s_{2 p}} d F\left(x_{1}, \ldots, x_{p}\right) d t_{1} \cdots d t_{p} \\
& =\int_{s_{11}}^{s_{21}} \cdots \int_{s_{1 p}}^{s_{2 p}} \prod_{k=1}^{p} g_{k}^{\prime}\left(x_{k}\right) S\left(x_{1}, \ldots, x_{p}\right) d x_{1} \cdots d x_{p} .
\end{aligned}
$$

Remark 2. Theorem 2 only holds for a lower or upper bounded variable. It is possible to extend it to unbounded variables. However, this involves the partition of a high dimensional space and the marginal cumulative distribution function or survival function which might be not easily obtainable. Further study is needed.

\section{Examples}

In this section, we give three examples when using the alternative expectation formulae to calculate the expectation is easier or more straightforward than standard approaches. In the first example, the mean of a continuous distribution can be directly calculated by the proposed formulae. In the second example, we apply the proposed formulae to obtain the form of the expectation of a singular distribution, and we use the Monte Carlo method to approximate the expectation. In the third example, we apply the proposed formula on a multivariate singular distribution and derive the expectation. 


\subsection{Univariate case: continuous distribution}

We consider a continuous random variable $X$ with a cumulative distribution function $F(x)=\sin (2 x)$. Let the support be $[0, \arcsin (\sqrt{2} / 2)]$ and consequently $F(\arcsin (\sqrt{2} / 2))=1$. In this toy example, we aim to estimate the mean of this distribution. By Theorem 1, the mean is obtained immediately:

$$
\mathbb{E}(X)=\arcsin \left(\frac{\sqrt{2}}{2}\right)-\int_{0}^{\arcsin \left(\frac{\sqrt{2}}{2}\right)} \sin (2 x) d x=\arcsin \left(\frac{\sqrt{2}}{2}\right)-\frac{1}{2} .
$$

Note that, the calculation of the mean by the standard method based on the probability density function involves the integration by parts.

\subsection{Univariate case: importance sampling for a singular distribution}

Due to the lack of the probability density/mass function, it is hard to approximate the expectation by a Monte Carlo method when directly drawing samples from a singular distribution is difficult. We show that this can be easily solved by using the alternative formulae when the cumulative distribution function is obtainable. The code to replicate results in this section is available in the GitHub ${ }^{1}$.

Monte Carlo method provides a way to estimate the expectation $\mathbb{E}(g(X))$, $X \sim F_{1}$ when this expectation is not analytically obtainable. If directly drawing samples from its distribution $F_{1}$ is difficult, we may use the importance sampling to draw samples from a different distribution $F_{2}$ since

$$
\mathbb{E}(g(X))=\int g(x) d F_{1}=\int g(x) \frac{d F_{1}}{d F_{2}} d F_{2} .
$$

\subsubsection{Cantor distribution}

Consider a random variable $X$ from the Cantor distribution whose support is the Cantor set within $[0,1]$, we aim to estimate its characteristic function. Since Cantor distribution does not have a probability density/mass function, importance sampling based on Eq. (5) is not feasible. However, its cumulative distribution function is the Cantor function which can be numerically calculated. By Theorem 1, we have

$$
\mathbb{E}\left(e^{i t X}\right)=e^{i t}-\int_{0}^{1} i t e^{i t x} F(x) d x .
$$

\footnotetext{
$1_{\text {https://github.com/MathBilibili/Alternative-Expectation-formulas }}$
} 
To estimate Eq. (6), we draw samples $\left\{x_{k}\right\}_{k=1, \ldots, n}$ from a uniform distribution $\mathrm{U}[0,1]$ and have the estimator

$$
\begin{aligned}
& \widehat{\mathbb{E}\left(e^{i t X}\right)}=e^{i t}-\frac{1}{n} \sum_{k=1}^{n}\left(i t e^{i t x_{k}} F\left(x_{k}\right)\right) \\
& =\cos (t)+\frac{t}{n} \sum_{k=1}^{n} F\left(x_{k}\right) \sin \left(t x_{k}\right)+i\left(\sin (t)-\frac{t}{n} \sum_{k=1}^{n} F\left(x_{k}\right) \cos \left(t x_{k}\right)\right) .
\end{aligned}
$$

We draw $10^{5}$ samples independently from a uniform distribution $\mathrm{U}[0,1]$ and the Eq. (7) is calculated for different values of $t$, where the value ranges from -6 to 6 with a step size of 0.25 . The real part and imaginary part are shown in Fig. 1. It is clear that the estimated value of the characteristic function is sufficiently accurate.
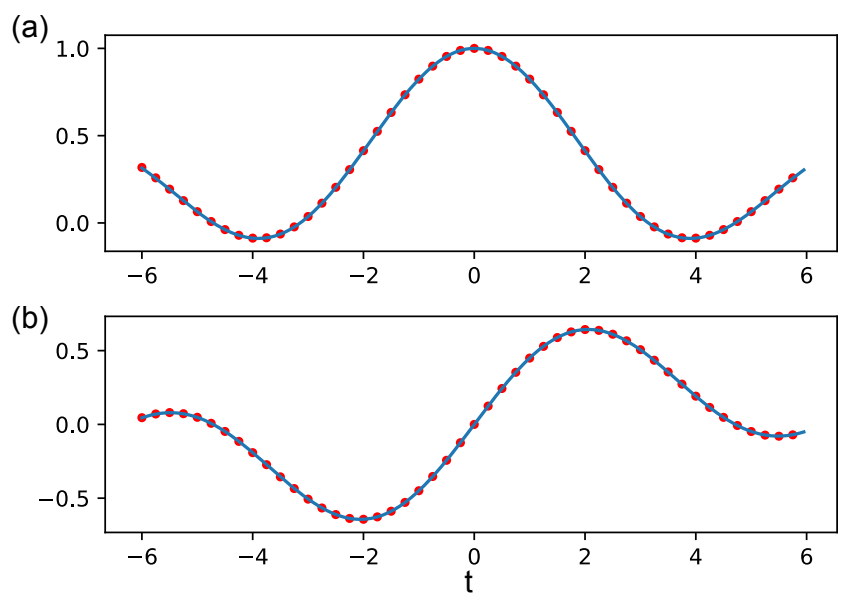

Fig. 1. The characteristic function and its estimates of Cantor distribution. Blue line is the true value, red dot is the estimated value obtained by Eq. (7). The real part and imaginary part are shown separately in panel (a) and (b).

\subsubsection{Dyadic expansion singular functions}

We consider the function studied by de Amo et al. (2012). Given a $x \in$ $[0,1]$, its dyadic expansion is $\sum_{n=0}^{\infty} 1 / 2^{m_{n}}$. For any $a \in(0,1)$ we denote

$$
F_{a}(x):=\sum_{n=0}^{\infty} a^{m_{n}-n}(1-a)^{n} .
$$


By the Proposition 2 of de Amo et al. (2012), $F_{a}(x)$ is an increasing function and satisfies

$$
\begin{aligned}
F_{a}\left(\frac{x}{2}\right) & =a F_{a}(x) ; \\
F_{a}\left(\frac{1+x}{2}\right) & =a+(1-a) F_{a}(x) .
\end{aligned}
$$

Since $F_{a}(0)=0$ and $F_{a}(1)=1, F_{a}(x)$ is a well defined cumulative distribution function. Theorem 2 of de Amo et al. (2012) shows that $F_{a}(x)$ does not admit non-zero derivative for all $a \in(0,1) \backslash\{0.5\}$. Therefore, $F_{a}(x)$ admits a singular distribution when $a \neq 0.5$. Meanwhile, it is clear that $F_{a}(x)=x$ when $a=0.5$.

We aim to estimate the mean of a random variable $X$ whose cumulative distribution function is $F_{a}(x)$, where $a \in\{0.15,0.5,0.85\}$. Although the probability density/mass function is not available when $a=0.15$ and 0.85 , we can nevertheless apply the alternative expectation formulae and the importance sampling to approximate the mean. We draw samples $\left\{x_{k}\right\}_{k=1, \ldots, 10^{4}}$ independently from a uniform distribution $\mathrm{U}[0,1]$. By Theorem 1 , the estimator is

$$
\widehat{\mathbb{E}(X)}=1-\frac{1}{10^{4}} \sum_{k=1}^{10^{4}} F_{a}\left(x_{k}\right) .
$$

The result is shown in Fig. 2.

\subsection{Multivariate case}

For the multivariate case, we consider a multivariate singular distribution inspired by Koopmans (1969). Given a lower bound (0,0,0) and an upper bound $(1,1,1)$, the cumulative distribution function of a multivariate random variable $(X, Y, Z)$ is defined as:

$$
F(x, y, z)= \begin{cases}2 x y, & x+y \leq z \\ 2 x z+2 y z-x^{2}-y^{2}-z^{2}, & z<x+y ; x \leq z ; y \leq z \\ 2 y z-y^{2}, & z<x+y ; z<x ; y \leq z \\ 2 x z-x^{2}, & z<x+y ; x \leq z ; z<y \\ z^{2}, & z<x ; z<y\end{cases}
$$

Since $F(0,0,0)=0 ; F(1,1,1)=1$ and $(\partial F) /(d x) \geq 0 ;(\partial F) /(d y) \geq 0 ;$ $(\partial F) /(d z) \geq 0,(X, Y, Z)$ is a well defined random variable. In addition, $\left(\partial^{3} F\right) /(d x d y d z)=0$ in $\mathbb{R}^{3}$. Hence, this distribution is a singular distribution. 


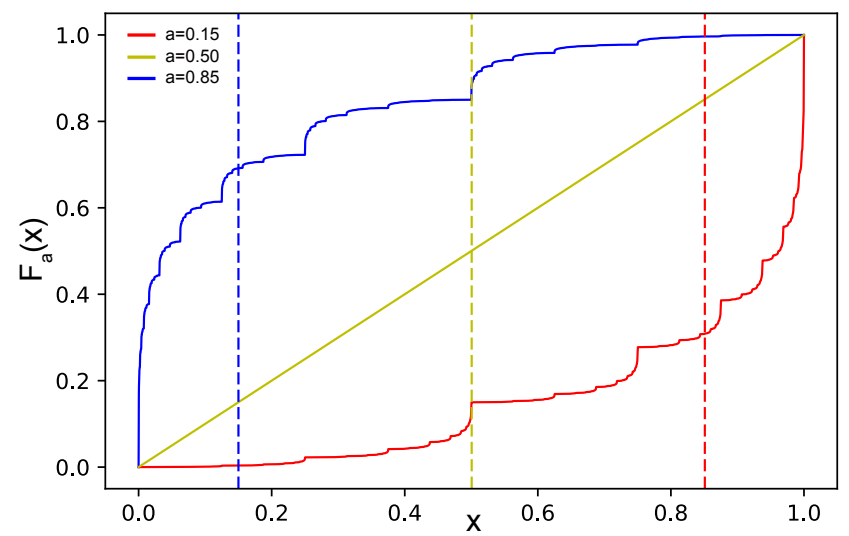

Fig. 2. The cumulative distribution function $F_{a}(x)$ and the estimated mean (indicated by the vertical dashed line), when $a=0.15$ (red), $a=0.5$ (yellow) and $a=0.85$ (blue).

We aim to calculate $\mathbb{E}((X-1)(Y-1)(Z-1))$. Since $F(x, y, z)$ is continuous at $(1,1,1)$, by Theorem 2 , it is straightforward to have

$$
\mathbb{E}((X-1)(Y-1)(Z-1))=-\int_{0}^{1} \int_{0}^{1} \int_{0}^{1} F(x, y, z) d x d y d z
$$

The integration is divided into five parts. We have

$$
\int_{0}^{z} \int_{0}^{x} 2 x y d y d x=\frac{z^{4}}{4}
$$

and

$$
\int_{0}^{z} \int_{x}^{z}\left(2 x z+2 y z-x^{2}-y^{2}-z^{2}\right) d y d x=\frac{z^{4}}{6}
$$

and

$$
\int_{z}^{1} \int_{0}^{z}\left(2 y z-y^{2}\right) d y d x=\frac{2}{3} z^{3}-\frac{2}{3} z^{4}
$$

and

$$
\int_{0}^{z} \int_{z}^{1}\left(2 x z-x^{2}\right) d y d x=\frac{2}{3} z^{3}-\frac{2}{3} z^{4}
$$

and

$$
\int_{z}^{1} \int_{z}^{1} z^{2} d y d x=z^{4}-2 z^{3}+z^{2}
$$


Plug Eq. (9-13) into Eq. (8), we have

$$
\mathbb{E}((X-1)(Y-1)(Z-1))=-\int_{0}^{1}\left(\frac{1}{12} z^{4}-\frac{2}{3} z^{3}+z^{2}\right) d z=-\frac{11}{60} .
$$

Note that to obtain this expectation using the proposed formulae, it is not necessary for $(X, Y, Z)$ to have a probability density/mass function.

\section{Conclusions}

In this paper, we give the alternative formulae for the expectation of a random variable in both univariate and multivariate cases. These formulae are useful to evaluate the expectation when the distribution is singular or the probability density function is not analytically obtainable. Our examples show the usage of these formulae on singular distributed variables and we hope that this study provides additional ways to evaluate the characteristic of random variables whose distributions belong to broader categories.

\section{Acknowledgements}

This work was supported by a Cambridge International Scholarship from the Cambridge Commonwealth, European and International Trust. The author thank the anonymous reviewers for their helpful comments.

\section{References}

de Amo, E., Daz Carrillo, M., Fernndez-Snchez, J., 2012. Singular functions with applications to fractal dimensions and generalized Takagi functions. Acta Applicandae Mathematicae 119, 129-148. doi:10.1007/ s10440-011-9665-z.

Chakraborti, S., Jardim, F., Epprecht, E., 2019. Higher-order moments using the survival function: The alternative expectation formula. The American Statistician 73, 191-194. doi:10.1080/00031305.2017.1356374.

Hong, L., 2012. A remark on the alternative expectation formula. The American Statistician 66, 232-233. doi:10.1080/00031305.2012.726934.

Hong, L., 2015. Another remark on the alternative expectation formula. The American Statistician 69, 157-159. doi:10.1080/00031305.2015. 1049710. 
Jones, M.C., 2019. Letter to the Editor. The American Statistician 73, 105-105. doi:10.1080/00031305.2018.1556736.

Koopmans, L.H., 1969. Some simple singular and mixed probability distributions. The American Mathematical Monthly 76, 297-299. doi:10.1080/ 00029890.1969 .12000204 .

Kwong, H.S., Nadarajah, S., 2018. Expectation formulas for integer valued multivariate random variables. Communications in Statistics - Theory and Methods 47, 5514-5518. doi:10.1080/03610926.2017.1395052.

Lo, A., 2017. Functional generalizations of hoeffdings covariance lemma and a formula for kendalls tau. Statistics \& Probability Letters 122, $218-226$. doi:https://doi.org/10.1016/j.spl.2016.11.016.

Lo, A., 2019. Demystifying the integrated tail probability expectation formula. The American Statistician 73, 367-374. doi:10.1080/00031305. 2018.1497541.

Nadarajah, S., Mitov, K., 2003. Product moments of multivariate random vectors. Communications in Statistics - Theory and Methods 32, 47-60. doi:10.1081/STA-120017799.

Ogasawara, H., 2020. Alternative expectation formulas for real-valued random vectors. Communications in Statistics - Theory and Methods 49, 454-470. doi:10.1080/03610926.2018.1543773.

Song, P., Tan, C., Wang, S., 2019. On the moment generating function for random vectors via inverse survival function. Statistics \& Probability Letters 145, 345 - 350. doi:https://doi.org/10.1016/j.spl.2018.10. 012.

Song, P., Wang, S., 2019a. A further remark on the alternative expectation formula. Communications in Statistics - Theory and Methods 0, 1-6. doi:10.1080/03610926.2019.1672743.

Song, P., Wang, S., 2019b. On the moment generating functions for integer valued random variables. Communications in Statistics - Theory and Methods 48, 5169-5174. doi:10.1080/03610926.2018.1508716. 